\title{
Effect of Environment on Viscous Flow in Inorganic Oxide Glasses
}

\author{
Joseph H. Simmons and Pedro B. Macedo* \\ Institute for Materials Research, National Bureau of Standards, Washington, D.C. 20234
}

(February 9, 1971)

\begin{abstract}
Results from viscosity and shear structural relaxation measurements conducted above the liquidliquid phase transition of a series of immiscible inorganic oxide glasses are analyzed. A model is proposed which relates the temperature dependence of the complex modulus and viscosity to the behavior of microstructure in the glass resulting from supercritical fluctuations in composition. It is suggested that the critical microstructure induces differences in local environment in the glass which in turn cause the appearance of distributions of relaxation times. The model is formulated using elementary fluctuation theory and the resulting equations are successfully compared to the data.
\end{abstract}

Key words: Critical point theories; immiscibility; phase transitions; structural relaxation; transport phenomena; viscous flow.

\section{Introduction}

The investigation of viscous flow in glass-forming liquids has been approached from a variety of directions. These can be classified into the empirical equation of Fulcher [1], ${ }^{1}$ the thermodynamic approaches of Gibbs [2], Turnbull and Cohen [3], and Goldstein [4], and the kinetic approaches recently characterized by consideration of the structural relaxation mechanisms [5-7]. In the case of inorganic oxide glasses, the investigations of structural relaxation have been undertaken by means of ultrasonic spectroscopy. Recently, ultrasonic [8] and viscosity [9] measurements on vitreous $\mathrm{B}_{2} \mathrm{O}_{3}$ have yielded evidence conflicting with the predictions of the thermodynamic approaches, and thus have indicated the need for systematic studies of viscous flow by structural relaxation in such glassformers [9]. Before examining this evidence, let us first introduce the concept of a distribution of relaxation times.

Viscous relaxation investigations by means of ultrasonic spectroscopy consist of studying the interaction of high frequency sound waves with matter as a function of the time of interaction. As the observation time, which is the inverse of the ultrasonic frequency, approaches the structural relaxation time, interaction between the flowing, or moving species and the sound waves leads to a frequency-dependent complex modulus. The frequency-dependent shear viscosity is given by the imaginary part of the shear modulus,

\footnotetext{
*Also at the Vitreous State Laboratory, Catholic University of America, Washington, D.C. 20017

1 Figures in brackets indicate the literature references at the end of this paper.

$G^{*}(\omega)$ as follows [10]:

$$
\eta^{\prime}(\omega)=\omega^{-1} \operatorname{Im}\left[G^{*}(\omega)\right]
$$

where $\omega$ is the angular frequency. The frequencydependent modulus may in turn be expressed in terms of a sum of relaxation functions. This implies that the stresses are additive. In the case of liquids having simple distributions of relaxation times, such as those in this paper, the relaxation phenomena may be characterized in terms of two independent parameters which are the width and the most probable value of time of any existing distribution of relaxation times. While the choice of additive stresses appears arbitrary at first, we will show later that it is consistent with the physical interpretation of our model. The viscosity is now written as follows:

$$
\eta^{\prime}(\omega)=G_{\infty} \int_{-\infty}^{\infty} \frac{\tau}{1+(\omega \tau)^{2}} g(\ln \tau) d \ln \tau
$$

where $G_{\infty}$ is the instantaneous shear modulus, $\tau$ is a relaxation time, and $g(\ln \tau)$ is known as the distribution of relaxation times. The "static" viscosity, $\eta_{s}$, is the zero-frequency limit of $\eta^{\prime}(\omega)$,

$$
\eta_{s}=G_{\infty} \int_{-\infty}^{\infty} \tau g(\ln \tau) d \ln \tau
$$

The instantaneous shear modulus and the distribution of relaxation times can be investigated by shear ultra- 
sonic spectroscopy provided the material is fluid enough to relax in a time scale comparable to the period of the ultrasonic waves, and one is equipped with instruments sensitive enough to detect the highly damped shear waves.

The evidence conflicting with the thermodynamic approaches first came from ultrasonic measurements [8]. Log-Gaussian distributions of relaxation times were found to represent the data well for vitreous $\mathrm{B}_{2} \mathrm{O}_{3}$. At high temperatures, when the static viscosity followed an Arrhenian behavior, it was seen that attributing a single relaxation time to the flow units was sufficient to account for the frequency dependence of the modulus. As the temperature was reduced, however, the shear viscosity departed from the Arrhenius curve, and a broadening distribution of relaxation times was required to fit the data. It was seen that the broadening occurred concurrently with the departure of the viscosity from Arrhenian behavior. Use of the Eyring Rate-Equation led to an analysis of the distribution of activation energies. The latter was found to be Gaussian and to broaden with decreasing temperature as did the distribution of relaxation times. The mean activation energy, however, did not change with temperature over the range of measurements. In conclusion, contrary to the assumption implicit in most phenomenological equations of the thermodynamic approaches, the non-Arrhenian behavior of the viscosity was not associated with an increase in the average activation energy with decreasing temperature, but rather with the appearance of a distribution of energies; some indeed higher, but others lower than the average. These lower activation energies could not be explained within the framework of existing viscosity theories.

Further support for these conclusions came from measurements of the viscosity of $\mathrm{B}_{2} \mathrm{O}_{3}$ in the range $10^{10}$ to $10^{14} \mathrm{P}$ [9], in which the viscosity appeared to revert to Arrhenian behavior at low temperatures. The authors could find no reasonable fits of this extended data by any of the viscosity theories, as the parameter $\left[\partial \ln \eta_{s} / \partial(1 / T)\right]=E_{\text {app }}$ representing an apparent activation energy, approached a temperatureindependent behavior near $T_{g}$, while all existing theories predicted a continued increase with decreasing temperature. In this case, a temperatureindependent apparent activation energy implies Arrhenian viscosity behavior. They thus concluded that the temperature dependence of the viscosity was not controlled by structural effects, such as free volume, and configurational entropy, but rather by some activation energy effects, represented by $\left[\partial \ln \eta_{s} / \partial(1 / T)\right]$. A successful theory would have to lead to a constant parameter $\left[\partial \ln \eta_{s} / \partial(1 / T)\right]$, or activation energy spectrum near $T_{g}$. It became apparent that a microscopic model was needed which could explain the appearance of a symmetric distribution of activation energies, and give physical significance to both the distribution of relaxation times and the temperature dependence of the viscosity.

In analyzing the origin of a spectrum of relaxation times, one cannot differentiate between the occurrence of the same nonexponential relaxation for all flow units, and the weighted sum of varying but exponential relaxation effects caused by a varying environment [11]. This problem was considered in the analysis of annealing experiments on several inorganic glasses $[12,13]$. In the case of a borosilicate crown glass [12] both volume relaxation and ionic conduction were measured. Of the various models present in the literature, only a modified version of Fröhlich's model [14] attributing the existence of a distribution of activation energies to a distribution of environments, could fit both sets of data consistently. In these measurements, it was found that if the distribution were represented by two relaxation times, the fast volume relaxation could be related to the fast electrical conductivity relaxation. The results implied that there are definite regions in these materials which are associated with the different relaxations.

The connection between a distribution of activation energies and a distribution of environments was postulated long ago by Fröhlich, but until recently, due to a lack of sufficiently detailed electron micrographs and high temperature x-ray instrumentation, no structure was observed in molten oxide glasses with known distributions of relaxation times. The model presented in the annealing investigations ruled out the possibility of nonexponential decays, but only indicated the possibility of applying some environmental model to the analysis of structural relaxation in inorganic oxide glasses. The problem in interpreting ultrasonic and other relaxation experiments arises from the fact that a distribution of relaxation times does not necessarily indicate any particular molecular relaxation process. As Goldstein [11] clearly suggested, calculation of a distribution of relaxation times in the analysis of a response function is only a mathematical transform and cannot carry physical significance by itself. One must first begin with a model for the molecular mechanisms for structural relaxation and then derive a resulting set or distribution of relaxation times. For this reason, we have chosen to investigate the viscous relaxation process in a series of inorganic oxide glasses with predictable distributions of environments and to attempt to analyze the results in terms of the related microstructure.

Microstructure in molten oxides can best be controlled by selective doping, or by approaching the critical point of an immiscible system. Since x-ray data or electron micrographs describing the structure of normal glasses are lacking, immiscible systems offer the best solution due to the possibility of specifying the temperature dependence of the structure from analysis of the supercritical composition fluctuations.

The immiscibility phase transition is quite widespread in oxide glasses. Liquid-liquid phase separations occur in such systems by changes in composition associated with phases of widely different viscosities. Phase separation at the concentration which has the highest transition temperature, $T_{c}$ (top of the immiscibility dome), occurs by means of a pseudo secondorder phase transition which is characterized by the continuity of the free energy and its first derivatives 
across the transition boundary. As a consequence, the thermodynamically unstable fluctuations which lead to demixing below $T_{c}$, do not end abruptly when one raises the temperature to the critical point. These fluctuations in fact extend far into the supercritical $\left(T>T_{c}\right)$ region, where they are thermodynamically metastable, with associated well defined wavelengths and lifetimes. The resulting transient domains have some structural relationship to the subcritical immiscible phases, and thus are characterized by large viscosity differences.

These supercritical composition fluctuations drastically change the environment of the flow species as the temperature is varied. Consequently, the presence of these fluctuations in composition is expected to have large effects on the viscous flow processes of the materials. Recently, light scattering and $x$-ray diffraction experiments have observed such fluctuations above the critical point $[15,16]$. The effect is usually referred to as critical opalescence.

Theoretical analyses first derived to describe gasliquid critical point phenomena, were applied to liquid-liquid phase transitions by replacing density fluctuations with fluctuations in composition [15].

Equations are thus available to describe the wavelengths, $\Lambda$, and lifetimes, $\tau_{f}$, of the existent fluctuations as a function of temperature for $T>T_{c}$. The wavelength, or range of fluctuations varies from very small values far from the critical point, to macroscopic extent near $T_{c}$. The range of fluctuations, $\Lambda\left(T / T_{c}\right)$, is expressed as:

$$
\Lambda=l /\left(T / T_{c}-1\right)^{1 / 2} .
$$

The classical exponent of $1 / 2$ is shown here, but there has been strong evidence that a larger value such as 0.6 may be more appropriate for some liquids. Such a small difference has little effect on our model, so we will follow the classical equation. The parameter $l$ is a constant of the material, and has been shown to vary little among liquid-liquid nonpolymeric systems (see table 4 of ref. [17]). Taking the average value of $11 \AA$, which also coincides with results from low angle $\mathrm{x}$-ray scattering measurements on $\mathrm{PbO}-\mathrm{B}_{2} \mathrm{O}_{3}-\mathrm{Al}_{2} \mathrm{O}_{3}$, we may plot the most probable fluctuation wavelength as a function of reduced temperature in figure 1 , and thus predict the size of the microstructure present in such critical systems above the solution temperature. It is now possible to investigate the effect of environmental microstructure on viscous flow by conducting structural relaxation measurements above the critical point of some immiscible oxide mixtures, and analyzing the resulting parameters in terms of reduced temperatures.

We have recently reported results from an experimental investigation of the behavior of the viscosity [18] and the frequency-dependent modulus [19] above the critical temperature of a series of immiscible oxide glasses. We will review, here, the salient features of these results and then proceed to analyze the observed supercritical effects in terms of a proposed mechanism for the interaction between the viscous flow process and the supercritical fluctuations in composition.

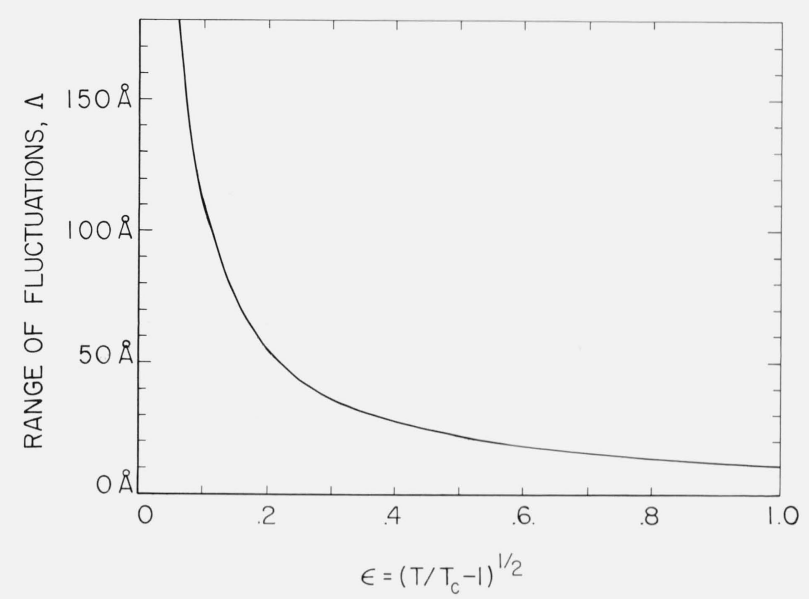

Figure 1. Fluctuation wavelength, $\Lambda$, plotted as a function of reduced temperature for $l=11 \mathrm{~A}$.

\section{Results from Viscous Relaxation Measure- ments on Critical Oxides}

The samples chosen for this study are four sodiumborosilicate oxides with similar high-temperature structures but widely varying critical temperatures. These characteristics were chosen to allow separation of effects due to the presence of supercritical composition fluctuations from the noncritical material behavior. Sample 1, with a composition of 70.5 percent $\mathrm{SiO}_{2}$, 22.7 percent $\mathrm{B}_{2} \mathrm{O}_{3}$, and 6.8 percent $\mathrm{Na}_{2} \mathrm{O}$ by mole is the critical composition (top of the dome) of its system [20], and has a critical temperature of $752^{\circ} \mathrm{C}$. Samples 2,3 , and 4 were made by adding 2.1 mol percent $\mathrm{CaO}$; 1.8 mol percent $\mathrm{Al}_{2} \mathrm{O}_{3}$; and 1.05 mol percent $\mathrm{CaO}+$ 0.9 mol percent $\mathrm{Al}_{2} \mathrm{O}_{3}$ respectively to the concentrations of sample 1 . Samples 2, 3, and 4 have resulting transition temperatures of 830,643 , and $741{ }^{\circ} \mathrm{C}$ respectively. These samples clearly provide many advantages. First, the viscosities of the separated phases differ by several orders of magnitude, so that the composition fluctuations can be expected to induce effects of large magnitude on the shear viscous relaxation parameters. Then, the critical temperatures of the samples occur at such high viscosities as to allow investigation of the distribution of shear relaxation times by ultrasonic relaxation spectroscopy.

The shear viscosity was measured by a rotation viscometer [21]. The results [18] were found to vary somewhat, between the samples, at high temperatures, reflecting the effect of doping (log viscosities of 2.41, $2.32,2.71$, and 2.46 at $1300^{\circ} \mathrm{C}$ for samples $1,2,3$, and 4 respectively). The viscosity curves were therefore normalized by the values at $1300{ }^{\circ} \mathrm{C}$, in order to study only the critical point effects. This normalized viscosity:

$$
\eta^{*}(T) \equiv \eta_{s}(T) / \eta_{s}\left(1300^{\circ} \mathrm{C}\right)
$$


was calculated for each sample, and is plotted against temperature in figure 2. It is evident from the figure

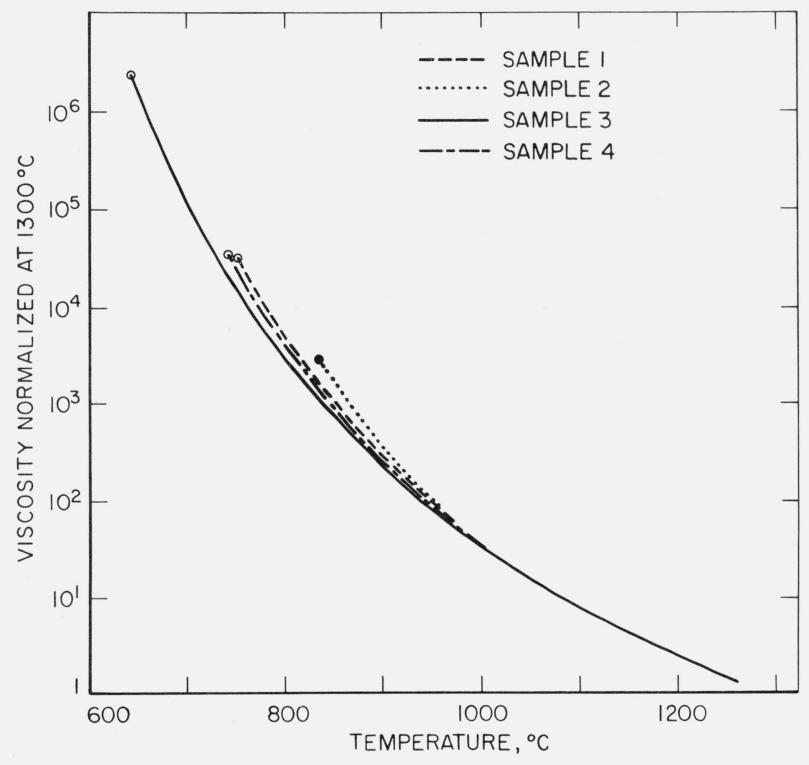

FigURE 2. Viscosity normalized by value at $1300{ }^{\circ} \mathrm{C}$ for the 4 samples.

The critical point of each sample is denoted by a circle on the appropriate viscosity curve.

that each curve departs from the curve of sample 3 as the respective critical points are approached from above. This indicates that the growing microstructure in the samples has a definite interaction with the viscous flow process. Further analysis of this excess viscosity was made possible by assuming that the noncritical viscosity behavior could be represented by the values of sample 3 since this sample had a critical temperature lower than the others (samples 1,2, and 4) by 106,187 , and $98{ }^{\circ} \mathrm{C}$ respectively. A normalized excess viscosity was then defined as follows:

$$
\Delta \Phi(T) \equiv \Delta \eta^{*}(T) / \eta_{0}^{*}(T)=\left[\eta^{*}(T)-\eta_{0}^{*}(T)\right] / \eta_{0}^{*}(T)
$$

where the noncritical viscosity, $\eta_{0}^{*}(T)$, is represented by the viscosity of sample 3 . The normalized excess viscosity is shown in figure (3) plotted against reduced temperature: $\left(T / T_{c}-1\right)^{1 / 2}$. It is seen that when the excess viscosity $\Delta \eta^{*}(T)$ is normalized by the noncritical viscosity, $\eta_{0}^{*}(T)$, the result, $\Delta \Phi(T)$, has the same reduced-temperature behavior for all samples. The supercritical excess viscosity reached a value of twice the noncritical viscosity $\left(\Delta \eta^{*} \approx 2 \eta_{0}^{*}\right)$ near the transition temperature, but it should be noticed that there are no indications of a divergence down to $T / T_{c} \approx 1.0009$.

Ultrasonic relaxation measurements were also conducted on the same series of glasses to investigate the distributions of relaxation times, and the parameters which control the viscous flow [19]. The measurements showed that the isothermal frequency dependence of the moduli could be best represented

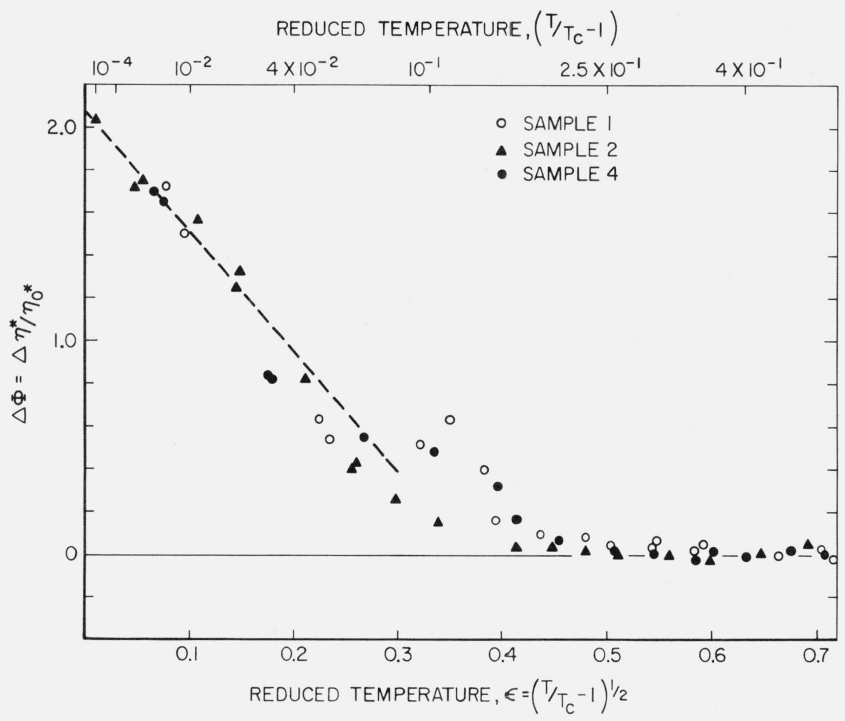

FigURE 3. Normalized excess viscosity plotted as a function of reduced temperature.

The solid horizontal line represents sample 3 .

by $\log$-Gaussian distributions of relaxation times down to temperatures close to $T_{c}\left(T / T_{c} \approx 1.0045\right)$.

Substitution of the structural relaxation distributions so determined into the equation calculating static viscosity, eq (3), yields values within 10 percent of the measured static viscosity. Since this is within the experimental accuracy of the ultrasonic results, we concluded that no additional relaxation process occurring at frequencies lower than the structural relaxation was present in the frequency-dependent description of the shear modulus and viscosity. Such a low frequency process (mode-mode coupling) has been proposed $[22]$ to account for the supercritical excess viscosity and is seen to be inapplicable to the systems studied herein. Therefore, the entire critical-point effect on the shear flow mechanism is contained in the structural relaxation processes rather than in some separate interaction process such as proposed [22] by the mode-mode coupling models. We have shown however, in a separate publication [23], that a low frequency process similar to the mode-mode coupling occurs in the volume relaxation mechanism of sample 1 .

In order to further investigate the effect of the static microstructure on the relaxation and viscous processes we must consider the relationship between the viscosity and the parameters obtained in the ultrasonic spectroscopy. Since the structural distribution of relaxation times $[g(\ln \tau)$ in eq 2] was found to be log-Gaussian, the static viscosity may be written as:

$$
\begin{aligned}
\eta_{s}(0) & =\operatorname{limit}_{\omega \rightarrow o}\left\{G _ { \infty } \int _ { - \infty } ^ { \infty } \left(\frac{\tau}{1+(\omega \tau)^{2}} \frac{d \ln \tau}{(2 \pi \Theta)^{1 / 2}} \exp \right.\right. \\
{\left.\left.\left[-\ln ^{2}\left(\tau / \tau^{\prime}\right) /(2 \Theta)\right]\right)\right\} } & \\
& =G_{\infty} \bar{\tau}=G_{\infty} \tau^{\prime} \exp (\Theta / 2)
\end{aligned}
$$


where $\bar{\tau}$ is the average and $\tau^{\prime}$ the most probable relaxation times, and $\theta$ is the width or variance $\left[\Theta \equiv<(\Delta \ln \tau)^{2}>\right]$ of the distribution.

Further analysis of the ultrasonic data yielded the behavior of the three parameters which control the shear viscosity. These are the instantaneous shear modulus, $G_{\infty}$ the most probable relaxation time, $\tau^{\prime}$, and the width of the distribution of relaxation times, $\Theta$, as per eq (7). The first two parameters, $G_{\infty}$ and $\tau^{\prime}$, did not appear to be subject to critical point effects and thus seem to be independent of the change in size of the microstructure as $T_{c}$ is approached. It was found, however, that the width of the distribution of relaxation times was greatly affected by the approach of the transition temperature, and thus by the changing microstructure. As shown in figure 4, the samples have narrow distributions at high temperatures where the fluctuations are small. The distributions then broaden drastically as the temperature is lowered to the phase transition. Finally, the broadening saturates as the width appears to approach a finite value close to $T_{c}$, where the fluctuations have grown to macroscopic sizes. The large increase in the width is related to the viscosity anomaly and represents some interaction between the composition fluctuations and the shear viscous relaxation process. The saturation of this broadening very close to $T_{c}$ indicates that the interaction is weakening when $T_{c}$ is approached and the fluctuation wavelengths are large.

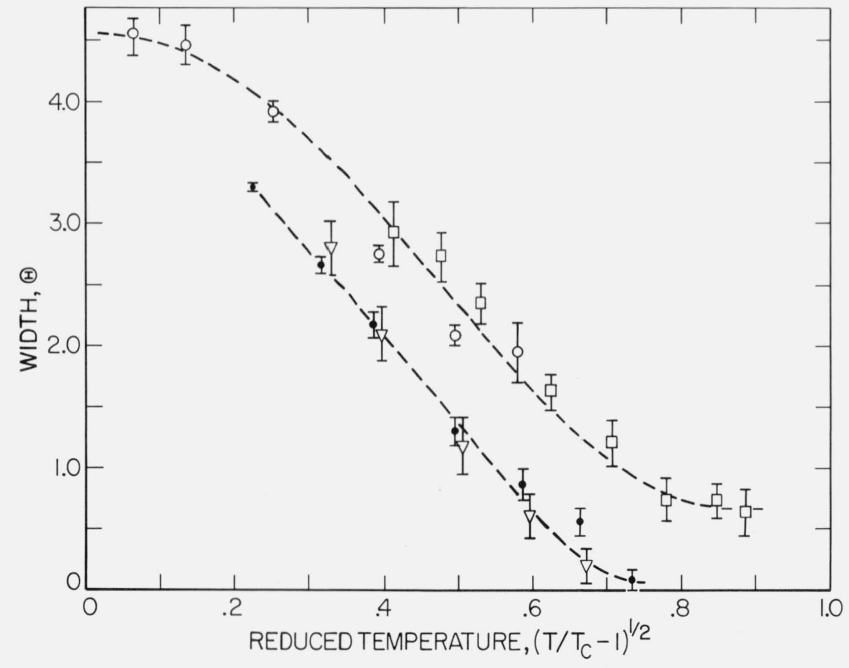

Figure 4. Width of the distribution of relaxation times for sample 1 (solid circles), sample 2 (open circles), sample 3 (squares), and sample 4 (triangles) as a function of reduced temperature. The curves were drawn to emphasize the general behavior of the materials.

\section{Analysis of the Environmental Relaxation Model}

\subsection{Deveiopment of the Model}

In this discussion of the model we will be concerned with presenting physical arguments followed by a mathematical formulation of the behavior of the distributions of relaxation times and activation energies in terms of the appearance and growth of local microstructure in the melt. These distributions, therefore, will be related to a distribution of the molecular environments. The latter is then to be estimated in terms of the supercritical probability for the occurrence of composition fluctuations in a local region defined in the model by the range of forces controlling structural relaxation of the flow units.

The results from the supercritical, shear relaxation experiments indicate that while the distribution of relaxation times is narrow at high temperatures, a large broadening occurs as the critical point is approached from above. Figure 4 shows that all four curves follow the same general behavior within the range of measurements, despite the vastly different critical temperatures of the oxide mixtures, when the width is analyzed in terms of the reduced temperature.

Recent analysis of measurements of the volume structural relaxation properties [23] of sample 1 resulted in the determination of the average concentration fluctuation lifetime, $\bar{\tau}_{f}$, whose magnitude is compared in figure 5 with the most probable volume, $\tau_{v}^{\prime}$, and shear, $\tau_{s}^{\prime}$, relaxation times. While $\tau_{v}^{\prime}$ approaches the concentration fluctuation lifetimes at high temperatures, the shear distribution centered at $\tau_{s}^{\prime}$ is always shorter than $\bar{\tau}_{f}$. The shear structural relaxation process, therefore, occurs in an apparently static environment, and the composition fluctuations only interact with the

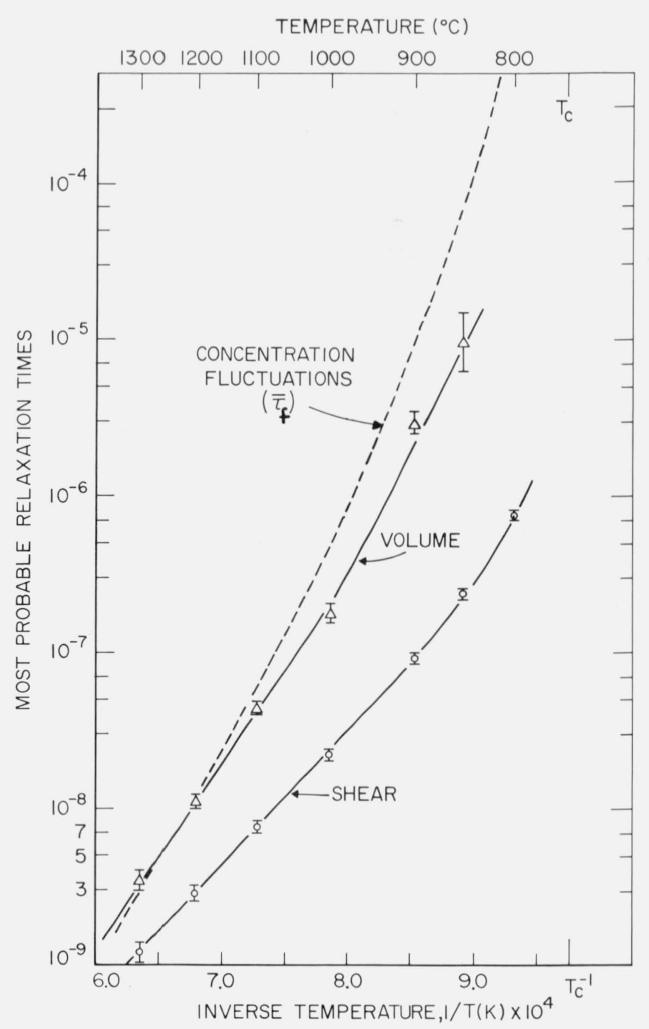

FIGURE 5. Average concentration fluctuation lifetime compared to the most probable volume and shear relaxation times (Reprinted from Ref. [23]). 
shear flow process through the resulting temperaturedependent distribution of local environments.

A molecule rearranges its position to respond to a stress in a manner which is strongly dependent upon its environment. The large increase in the width of the distribution of relaxation times therefore could be related to the change in the local configuration of the material resulting from the supercritical fluctuations in composition. Furthermore, the broadening in the distribution of relaxation times then would result from the change in the distribution of local environments. Since it has been demonstrated that there is no direct mode-mode coupling between the shear relaxation mechanisms and the fluctuations in concentration, the flow process does not exchange energy with the fluctuations and only samples the presence of the resulting microstructure. The problem therefore reduces to the analysis of structural relaxation occurring in a temperature-dependent but quasi-static environment, because the concentration fluctuation times are longer than the shear structural relaxation times at all temperatures.

Assuming a direct relationship between distributions of environments and relaxation times, we may analyze the effect of the critical microstructure. A system will exhibit a single relaxation time if all its molecules or flowing species have equivalent neighborhoods. As a number of different configurations become available to the molecules, the relaxation process will vary spatially, and a distribution of times will appear which broadens as either the number of configurations or the variation of relaxation times among the neighborhoods increases. It is clear from our results that these two types of behavior are evident in our distribution of relaxation times as $T_{c}$ is approached. However, as the fluctuations grow to macroscopic sizes, we see that the number of configurations increases, becoming very large as the material phase-separates. The resulting divergence is however inconsistent with our results.

We note that a relaxing species cannot be subject to the nature of its environment without limit. As intermolecular forces have ranges, so must the relaxation mechanism. Thus, we postulate that the relaxation mechanism of a molecule is further characterized by a range (which we will call $r_{0}$ ) within which the number of configurations affect the relaxation time of the molecule. Changes in the number of configurations outside this range will be considered irrelevant in determining the relaxation time of the molecule. This concept results in a saturation of the broadening of the distribution very close to $T_{c}$, as the flowing species are insensitive to further changes in the environment. The magnitude of $r_{0}$ is characteristic of the relaxation process in a particular material, and is independent of critical phenomena. While it is quite possible that $r_{0}$ varies with temperature, in order to maintain the undetermined parameters to a manageable number, we will assume that its magnitude remains constant over the range of our measurements. This assumption is well justified since the changes in $r_{0}$ are expected to be small in the temperature range of interest to us, and the introduction of a finite range for the effective environment in structural relaxations carries a far greater effect than changes in the range itself. In order to formulate the relationship between the composition fluctuations and the distribution of relaxation times, let us turn to elementary fluctuation theory.

\subsection{Formulation of the Model}

The supercritical state of liquid mixtures is characterized by fluctuations in the local composition of the material. Since we are interested in the analysis of the width of a distribution of relaxation times in terms of composition fluctuations, we will consider the width, $\Theta=\left\langle(\Delta \ln \tau)^{2}\right\rangle$, as a dependent function of the mean-square composition change, $\left\langle(\Delta c)^{2}\right\rangle[24]$. Thus, we let the width of the relaxation time spectrum be dependent upon the fluctuations in local composition as follows:

$$
\ln \tau=\ln \tau_{0}+c(\partial \ln \tau / \dot{\partial} c) .
$$

This expression implies that $(\partial \ln \tau / \partial c)$ has been chosen to be independent of $\tau$ and $c$ with the result:

$$
\Theta=\left\langle(\Delta \ln \tau)^{2}\right\rangle=(\partial \ln \tau / \partial c)^{2}\left\langle(\Delta c)^{2}\right\rangle .
$$

This formulates the connection between the criticalpoint related changes in the distribution of relaxation times and the environmental changes brought about by the supercritical microstructure.

Assuming that the probability density for composition fluctuations is a normal or Gaussian function, then it follows from eqs (8) and (9) that the corresponding distribution of relaxation times is a logGaussian function of time of the form:

$$
\begin{gathered}
g(\ln \tau)=\left[2 \pi\left(\frac{\partial \ln \tau}{\partial c}\right)^{2}\left\langle(\Delta c)^{2}\right\rangle\right]^{-1 / 2} \\
\exp \left[-\frac{(\Delta \ln \tau)^{2}}{2\left(\frac{\partial \ln \tau}{\partial c}\right)^{2}}\left\langle(\Delta c)^{2}\right\rangle\right]
\end{gathered}
$$

Since the ultrasonic measurements have shown that $g(\ln \tau)$ is indeed a Gaussian in $(\ln \tau)$ in the critical oxide mixtures, then the choice of $\left\langle(\Delta \ln \tau)^{2}\right\rangle$ rather than another time expression such as $\left\langle(\Delta \tau)^{2}\right\rangle$ for the dependent function of eq (8) is justified. The assumption that $(\partial \ln \tau / \partial c)$ is independent of $\tau$ and $c$, at any temperature, therefore leads to a distribution consistent with the measured function $g(\ln \tau)$.

The change in the number of available configurations for relaxation resulting from supercritical fluctuations is written as the change in the mean-square composition fluctuation. The latter represents the average deviation of local composition in the supercritical mixture from the composition in a similar but homogeneous system. This is obtained by integrating the supercritical correlation function, $G(r)$, over the local volume, $V_{0} . G(r)$ is the increased probability above average of finding two particles of the same component at a distance $r$ apart and thus gives a measure of the 
probability of having deviations from the homogeneous composition [24].

$$
G(r)=\frac{1}{c_{0}^{2}}\left\langle\left[c\left(r_{1}\right)-\overline{c\left(r_{1}\right)}\right]\left[c\left(r_{2}\right)-\overline{c\left(r_{2}\right)}\right]\right\rangle-\delta\left(r_{1}-r_{2}\right)
$$

$c_{0}$ is the average density of the component of interest, the subscripts 1 and 2 represent two locations in space at $r_{1}$ and $r_{2}$, separated by a distance $r$, and the averaging is a space average. $\delta\left(r_{1}-r_{2}\right)$ is a "delta" function expressing the certainty of finding a particle at $r_{2}$ when $r_{2}=r_{1}$. The supercritical pair correlation function, $G(r)$, varies from zero, far from the critical point, where no composition fluctuations are present, to one at the critical point.

The supercritical deviation in local composition may now be calculated by integrating the clustering or ordering probability represented by $G(r)$ over a local volume, defined by a cutoff function $f(r)$.

$$
\left\langle[\Delta c]^{2}\right\rangle_{f}=\int G(r) f(r) d V / \int f(r) d V
$$

where the local composition deviation is represented by $\left\langle[\Delta c]^{2}\right\rangle_{f}$ in order to indicate that it is associated with the cutoff function $f(r)$. The screening function represents the limitation of the range of interaction in the structural relaxation process, and thus is used to limit the integration to a local volume. As discussed earlier, this cutoff or screening function, $f(r)$, is necessary here, since the ultrasonic and viscosity results show a saturation in the interaction of the viscous relaxation process with the composition fluctuations at temperatures close to the critical where the fluctuations grow to macroscopic sizes. The specific form of this function cannot be derived at this time, since the microscopic mechanism of structural relaxation is not known. In earlier papers [25, 17], the authors denoted $f(r)$ by a step function:

$$
f(r)=\left\{\begin{array}{l}
1 \text { for } r<r_{0} \\
0 \text { for } r>r_{0} .
\end{array}\right.
$$

However, in order to take into account the gradual decrease in influence as the distance from the flow unit is increased, a Gaussian screening function is used herein.

$$
f(r)=\exp \left(-r^{2} / 2 r_{0}^{2}\right) .
$$

Little difference results from this choice, except that the final equation may be solved in closed form. The range of this function is $r_{0}$, and events which occur at $r>r_{0}$ have little effect on the behavior of the flow unit at the center.

The choice of a functional form for the pair correlation function, $G(r)$, presents a further problem. A popular form, to date, has been the Ornstein-ZernikeDebye (OZD) pair correlation function [24]:

$$
G(r)_{\mathrm{OZD}}=k_{B} T \alpha_{0} \frac{e^{-r / \Lambda}}{r}
$$

where $\Lambda$ is the range of interaction and $\alpha_{0}$ is a material constant. The Fourier transform of this expression has had some success with light scattering [15], and low angle $\mathrm{x}$-ray scattering [16] at small wave numbers, $k$, and is written as:

$$
g(k)_{\mathrm{OZD}}=\alpha_{0 k} k_{B} T /\left(a+b k^{2}\right) .
$$

The OZD pair correlation function is applicable only at large distances since the validity of the Fourier transform in eq (16) is limited to small $k$. As a result, $G(r)_{\text {OzD }}$ has an unacceptable divergence at the origin, and cannot represent the critical correlation function at small distances. This failure of the OZD function to suitably represent the supercritical correlation function has been demonstrated in various $\mathrm{x}$-ray measurements [16, 26] for large $k$-values, as the inverse intensity departs drastically from a $k^{2}$-dependence (eq (16)). Following our model, we see that at temperatures close to the critical point, the correlation length $\Lambda$ is of the order of $r_{0}$, and the integration of eq (12) weighs heavily the intermediate portion of the pair correlation function. It has been shown in a previous paper [25] that, as a result, the OZD function fails to describe adequately the data near $T_{c}$. In order to improve this present model let us choose a function which satisfies the same asymptotic conditions as the OZD function for small $k$ and large $r$, and has a better behavior where the latter fails at large $k$ and intermediate distances. The conditions which must be met are that the Fourier transform of the correlation function follow a $k^{2}$-dependence at low $k$-values, and that the function be finite at the origin $(r=0)$. The well-known Gaussian function satisfied these requirements.

$$
G^{\prime}(r)=\alpha_{0}^{\prime} k_{B} T \exp \left(-r^{2} / 2 \Lambda^{2}\right)
$$

with a Fourier transform:

$$
g^{\prime}(k)=\alpha_{0 k}^{\prime} k_{B} T \exp \left(-k^{2} / 2 k^{2}\right)
$$

where $\kappa=1 / \Lambda$. The Fourier transform of the Gaussian expression may be expanded into a series at small wave numbers:

$$
\operatorname{limit}_{k \rightarrow 0} g^{\prime}(k)=\alpha_{0 k}^{\prime} k_{B} T /\left[1+\frac{1}{2}(k / \kappa)^{2}+\frac{1}{4}(k / \kappa)^{4}+\ldots .\right]
$$

thus having the same asymptotic behavior $\left(a+b k^{2}\right)$ as the OZD function. Furthermore, the additional positive $k^{4}$-term which appears for larger $k$-values is consistent with an upward bend in the intensity curves $\left(I^{-1}\right.$ versus $\left.k^{2}\right)$ resulting from low-angle $\mathrm{x}$-ray scattering measurements. In addition, the Gaussian pair-correlation function remains finite at the origin, and therefore can be normalized to one-a necessary property since the correlation function is a probability density. We have chosen this particular form for the correlation function only as a means of analyzing our results. Although the Gaussian function appears to satisfy a number of conditions for suitability, since we cannot 
formulate the functional form of the screening function, $f(r)$, from microscopic considerations, we by no means intend to propose that agreement of the model with data should suggest the Gaussian expression as a new universal supercritical correlation function.

The local deviation in composition is now given by the integration of the pair-correlation function as per eq (12).

$$
\left\langle(\Delta c)^{2}\right\rangle_{\text {Gauss }}=\alpha_{0}^{\prime} k_{B} T\left[\frac{\Lambda^{2}}{\Lambda^{2}+r_{0}^{2}}\right]^{3 / 2} .
$$

Defining the ratio of the structural relaxation range, $r_{0}$, to the correlation length, $\Lambda$, by $\mathscr{R}$, and using the temperature dependence of $\Lambda$ discussed earlier,

$$
\begin{aligned}
\mathscr{R} & \equiv r_{0} / \Lambda=\left(r_{0} / l\right)\left(T / T_{c}-1\right)^{1 / 2} \\
& =\mathscr{R}_{0}\left(T / T_{c}-1\right)^{1 / 2}
\end{aligned}
$$

we may write the local deviation in composition in terms of reduced temperature:

$$
\left\langle(\Delta c)^{2}\right\rangle_{\text {Gauss }}=\alpha_{0}^{\prime} k_{B} T\left[\frac{1}{1+\mathscr{R}_{0}^{2}\left(T / T_{c}-1\right)}\right]^{3 / 2} .
$$

The expression is now inserted into eq (9) and once a suitable value of $(\partial \ln \tau / \partial c)$ is obtained, the distribution of relaxation times or activation energies may be expressed in terms of the reduced temperature.

\section{Calculations}

The factor $(\partial \ln \tau / \partial c)$ presents the final problem. Throughout the paper, we have discussed the relaxation properties in terms of the natural logarithm of time, $\ln \tau$, instead of the time. A useful transform is accomplished by the Eyring Rate Equation.

Therefore:

$$
\tau=A e^{-S / k_{B}} e^{E / k_{B} T} .
$$

$$
\tau / \tau^{\prime}=\frac{A}{A}, e^{\left(E-E^{\prime}\right) / k_{B} T} e^{-\left(S-S^{\prime}\right) / k_{B}} .
$$

This transformation allows the use of "activation energy" distributions instead of distributions in $\ln \tau$. The activation energy format is used here for convenience and to calculate $(\partial \ln \tau / \partial c)$. We are not concerned with whether shear structural relaxation occurs by a simple rate process in the strict Eyring sense, in these glasses, although some indication to that effect is seen in the Arrhenian behavior of the most probable relaxation time at temperatures above $900{ }^{\circ} \mathrm{C}$.

Some data [27] is available on the relative behavior of the activation entropy and energy as a function of composition in a borosilicate system where $\mathrm{B}_{2} \mathrm{O}_{3}$ replaces $\mathrm{SiO}_{2}$. Since this process occurs during the phase separation of the oxides investigated herein, we will use the relative magnitudes of $\left(\frac{\partial E}{\partial c}\right)$ and $\left(\frac{\partial S}{\partial c}\right)$ to deduce the proper temperature dependence of $\left(\frac{\partial \ln \tau}{\partial c}\right)$ in our system, according to the following equation.

$$
\left(\frac{\partial \ln \tau}{\partial c}\right)=\frac{1}{A}\left(\frac{\partial A}{\partial c}\right)+\left(\frac{1}{k_{B} T}\right)\left(\frac{\partial E}{\partial c}\right)-\left(\frac{1}{k_{B}}\right)\left(\frac{\partial S}{\partial c}\right) .
$$

In order to compare the equation with the data [27] we combine the first and third terms:

$$
\left(\frac{\partial \ln \tau}{\partial c}\right)=\frac{1}{k_{B} T}\left(\frac{\partial E}{\partial c}\right)-\frac{1}{k_{B}}\left(\frac{\partial S^{*}}{\partial c}\right) .
$$

Measurements of viscosity at high temperatures in the Arrhenius temperature range yield both terms of eq (26). Figure 6 represents the temperature dependence of the subsequently calculated $\left(\frac{\partial \ln \tau}{\partial c}\right)$. The

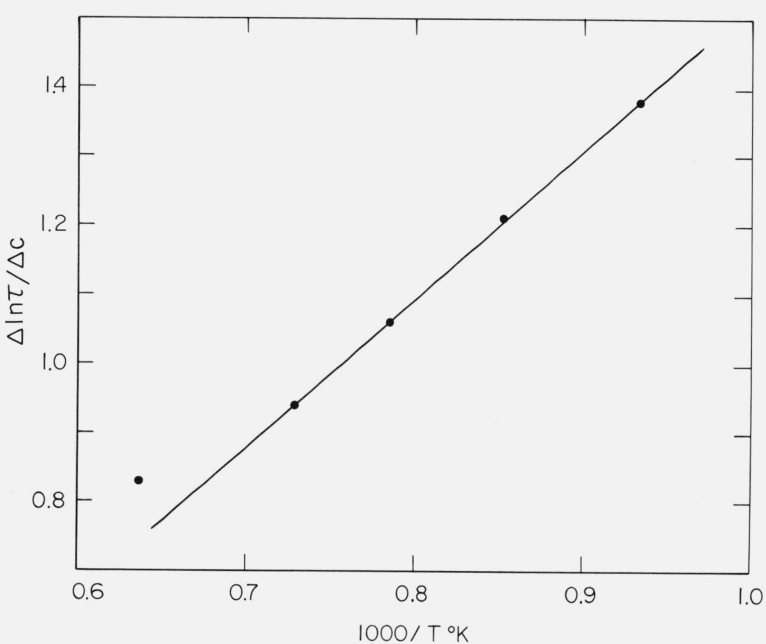

Figure 6. Plot of (J ln $\tau / \partial \mathrm{c})$ typical of the immiscible system studied here.

These points were calculated from the data of Ref. [27].

activation energy term appears to dominate in the temperature range of interest to us and the factor $\left(\frac{\partial \ln \tau}{\partial c}\right)$ is found to change inversely with temperature. Thus we may write:

$$
\left(\frac{\partial \ln \tau}{\partial c}\right)=\left[2.15\left(\frac{1000}{T}\right)-0.627\right] .
$$

The width of the relaxation time spectrum $g(\ln \tau)$ consequently becomes:

$$
\left\langle(\Delta \ln \tau)^{2}\right\rangle=\left\langle(\Delta E)^{2}\right\rangle /\left(k_{B} T\right)^{2} .
$$

$=\alpha_{0}^{\prime}\left[2.15\left(\frac{1000}{T}\right)-0.627\right]^{2}\left(k_{B} T\right)\left[\frac{1}{1+\mathscr{R}^{2}}\right]^{3 / 2}$ 
Before comparing the results with our model, we note from figure 4, that two of the samples investigated (samples 2 and 3) follow curves which appear to be shifted from the others. In the case of sample 3, there may be a high temperature residual width responsible for the shift. Since we are only interested in analyzing the broadening attributed to the change in structure resulting from the composition fluctuations, we subtract this width, and consider only the fractional change in width. A lack of high temperature data prevents a similar analysis of the data from sample 2, therefore, we will assume that it also has a residual width.

The data in the form:

$$
\begin{gathered}
\Delta=\left\langle(\Delta \ln \tau)^{2}\right\rangle\left\{\alpha_{0}^{\prime} k_{B} T\left[2.15\left(\frac{1000}{T}\right)-0.627\right]^{2}\right\}^{-1} \\
=\left[\frac{1}{1+\mathscr{R}^{2}}\right]^{3 / 2}
\end{gathered}
$$

is plotted in figure 7 for all four samples. The individual deviations from the composite curve are of the order of the experimental uncertainty, as can be seen from the error bars on the data. At this point, the equation proposed above can be fitted to the data, with $\alpha_{0}^{\prime}$ and $\mathscr{R}_{0}$ as undetermined variables. The results are shown in figure 7. The proposed function describes the three regions in the curve. Of particular interest are the large increase in width and the saturation of the width near $T_{c}$. Using the value of $11 \AA$ for $l$, the range of interaction for structural relaxation, $r_{0}$, is calculated to be $41 \AA$. In a previous paper, using the OZD function, we calculated $r_{0}$ to be $56 \AA$ [25]. This shows that while the form of the pair-correlation function influences the value obtained, its order of magnitude is well established by the data in relation to the three regions measured in $\left\langle(\Delta \ln \tau)^{2}\right\rangle$. Such a large value for $r_{0}$ indicates that shear viscous flow is truly a wide ranging process!

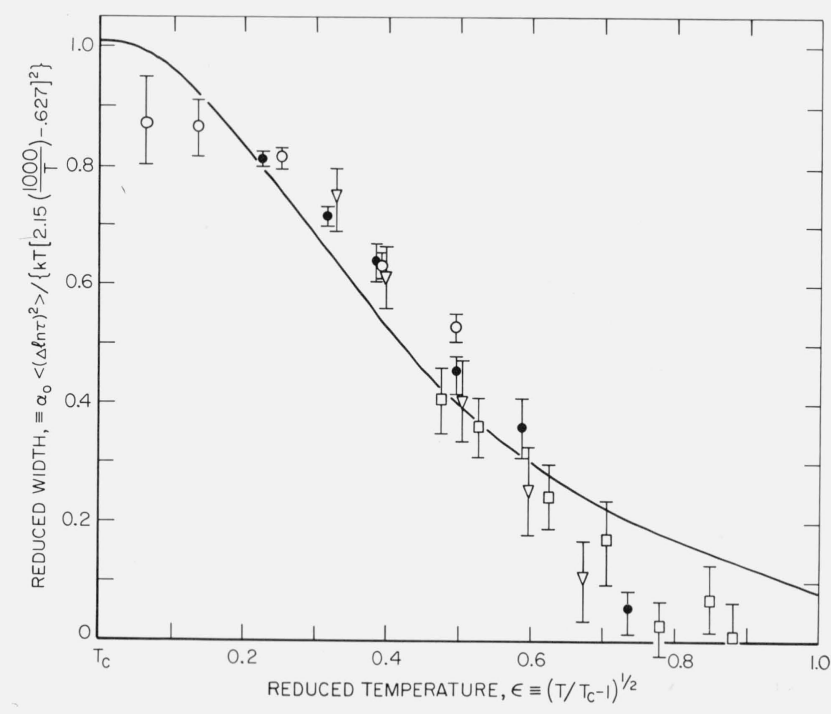

Figure 7. Comparison of the critical increase in width, with the proposed model, (solid line).

\section{Summary and Conclusion}

\subsection{Treatment of Supercritical Relaxation Experiments by the Environmental Relaxation Model}

A model is proposed which indicates that systems with a broad continuous distribution of mobilities will exhibit large supercritical effects. The model predicts that the supercritical effect will manifest itself in the width of the distribution of relaxation times. The relaxation mechanism is assumed to be characterized by a distance, $r_{0}$, which represents a finite range for the interactions which lead to relaxation. This range is characteristic of the material and independent of critical point phenomena. The dependence of width on reduced temperature, $\epsilon$, consists of three regions characterized by the relative sizes of $\Lambda$, the range of fluctuations, and $r_{0}$ the range of structural influence, as shown in figure 8 where we have represented the

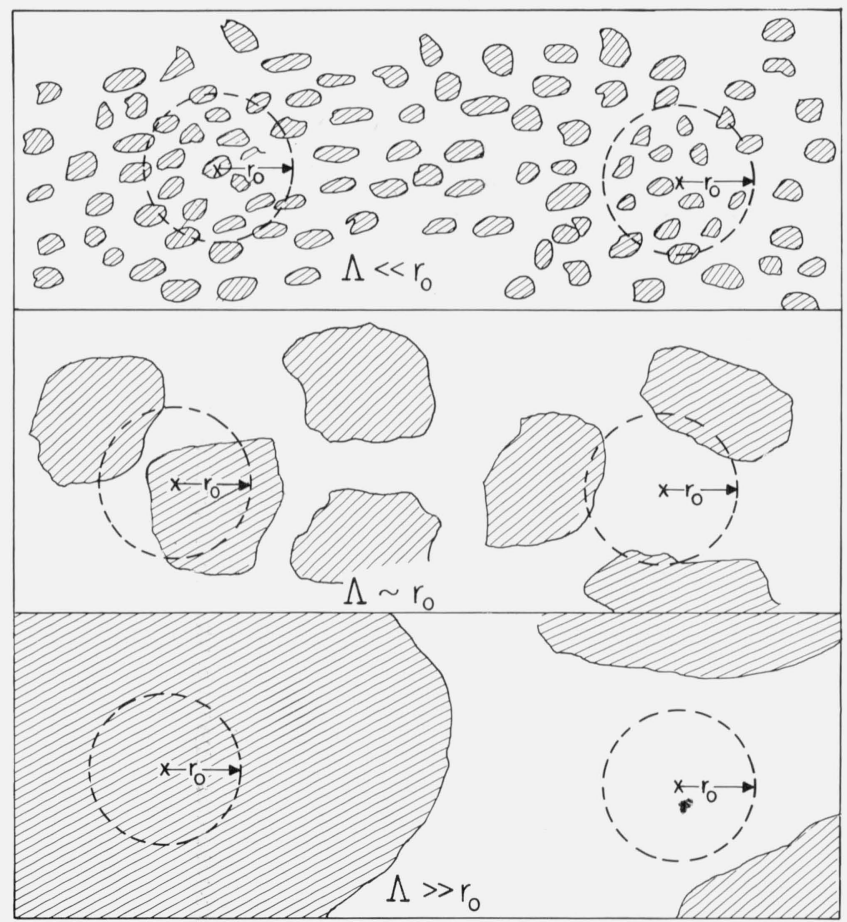

Figure 8. Typical microstructure expected to produce the three regions in the distribution of relaxation times, as a function of the relative sizes of $\mathrm{r}_{0}$ and $\Lambda(\epsilon)$.

distribution of mobilities by two distinct phases for simplicity. Region I, in which $\Lambda(\epsilon) \ll r_{0}$, represents high temperatures, far above $T_{c}$, and shows that due to space averaging within $V_{0}=4 / 3 \pi r_{0}^{3}$, all relaxing species interact with similar neighborhoods and the distribution of relaxation times is narrow, and in simple liquids is single. The distribution is temperature independent and Arrhenian viscosity behavior is observed. In Region II, as one approaches the critical temperature, $\Lambda$ approaches $r_{0}$. Now, large differences in environment appear as relaxing species begin to be surrounded by different local environments. The 
width of the relaxation time spectrum increases as the temperature approaches the critical point and the viscosity no longer follows Arrhenian behavior. An anomalous excess viscosity appears. In Region III, $\Lambda(\epsilon) \gg r_{0}$, the temperature is close to the critical point, and the fluctuations are large. Relaxing species are well imbeded within the fluctuations so that further changes in $\Lambda(\epsilon)$ have little effect on the local compositions. The width of the relaxation time spectrum saturates, changing little as one approaches $T_{c}$. This is reflected in a saturation of the anomalous excess viscosity despite a divergence of the correlation length $\Lambda$. The viscosity appears to revert to Arrhenian behavior because, although a broad distribution of relaxation times remains at temperatures near $T_{c}$, the character of the distribution ceases the change with temperature that gave rise to the non-Arrhenian behavior. Actual Arrhenian behavior is however only observed if the most probable relaxation time, $\tau^{\prime}$, is also Arrhenian. The Gaussian function proposed fits all three regions well and has the proper asymptotic behavior near and far from $T_{c}$. The range of structural influence, $r_{0}$, is quite large with a value of 41 to $56 \AA$, and spans over many molecular diameters. This explains why, in some oxide glasses, the addition of small amounts of impurities changes the relaxation properties: an addition of $5 \mathrm{ppm}$ of $\mathrm{Na}_{2} \mathrm{O}$ to pure $\mathrm{SiO}_{2}$ changes the viscosity by a factor of two [28].

\subsection{Generalization to Other Materials}

The work presented herein has been concerned with explaining the origin of distributions of relaxation times observed above the critical points of a series of immiscible oxide mixtures. For this reason, we have been primarily concerned with describing the microstructure in the environment in terms of supercritical composition fluctuations. Microstructure in the environment may, however, arise from other causes: association, density and various other ordering phenomena. The analysis presented here would also apply in such cases in qualitative terms. The three regions described previously would be expected to occur in an analysis of the spectral width as the order parameter varies in size relative to the range of structural influence, $r_{0}$.

Boron trioxide glass, for example, has a log-Gaussian shear distribution of relaxation times [8] but has no composition fluctuations. Yet its relaxation time spectrum and its distribution of activation energies broaden as the temperature is lowered, exhibiting not only higher activation energy values as expected, but also lower ones as well. This apparent paradox is easily explained by the present relaxation model, as we attribute the appearance of lower and higher activation energies to the formation of a distribution of regions in the melt with varying degrees of association, some low and some high (low E and high E). A temperature dependent spectrum of relaxation times also occurs in $\mathrm{GeO}_{2}$ glass at temperatures near its transformation region [13]. According to the model, this would indicate that there is a degree of microheterogeneity whose size is of the order of $r_{0}$ at the transformation region in this glass. Since $r_{0}$ is a property of the material, it is likely to vary little among simple oxide glassformers so that the model suggests a value of $50 \AA$ to the size of the microstructure in $\mathrm{GeO}_{2}$ in the transformation region. Electron micrographs on $\mathrm{GeO}_{2}$ [29] have revealed that, indeed, there is microheterogeneity in $\mathrm{GeO}_{2}$ in the transformation region of the order of 25 to $50 \AA$.

Although, the environmental relaxation model can give a qualitative description of the behavior of the width of the relaxation time spectrum in terms of the size of the order parameter (i.e., Regions I, II, and III), a description in terms of temperatures is not possible for normal glasses. An analysis of the temperature dependence of the order parameter, and the form of the pair correlation function are needed before a treatment similar to the immiscible melts can be carried out on normal or single component molten oxides.

\section{References}

[1] Fulcher, G. S., J. Am. Ceram. Soc. 8, 339 (1925).

[2] Gibbs, J. H., Modern Aspects of the Vitreous State, Vol. 1, J. D. Mackenzie, Ed. (Butterworths, London, 1960).

[3] Turnbull, D., and Cohen, M. H., J. Chem. Phys. 34, 120 (1961).

[4] Goldstein, M., J. Chem. Phys. 51, 3728 (1969).

[5] Litovitz, T. A., Non-Crystalline Solids, V. D. Frechette, Ed. (John Wiley, New York, 1960).

[6] Montrose, C. J., and Litovitz, T. A., J. Acous. Soc. Am. 47, 1250 (1970).

[7] Isakovich, M. A., and Chaban, I. A., Soviet Phys. Doklady 10, 1053 (1966); Sov. Phys. JETP 23, 893 (1966).

[8] Tauke, J. Litovitz, T. A., and Macedo, P. B., J. Am. Ceram. Soc. 51, 158 (1968).

[9] Macedo, P. B., and Napolitano, A., J. Chem. Phys. 49, 1887 (1968).

[10 1 Herzfeld, K. F., and Litovitz, T. A., Absorption and Dispersion of Ultrasonic Waves (Academic Press, New York, N.Y., 1959).

[11] Goldstein, M., Modern Aspects of the Vitreous State, Vol. 3, J. D. Mackenzie, Ed. (Butterworth, Washington, 1964).

[12] Macedo, P. B., and Napolitano, A., J. Res. NBS 71A, 231 (1967).

[13] Napolitano, A., and Macedo, P. B., J. Res. NBS 72A, 425 (1968); Boesch, L., Napolitano, A., and Macedo, P. B., J. Am. Ceram. Soc. 53, 148 (1970)

[14] Fröhlich, H., Theory of Dielectrics (Clarendon Press, Oxford, 1949).

[15] Chu, B., J. Am. Chem. Soc. 86, 3557 (1964); Fisher, M., J. Math. Phys. 5, 944 (1964).

[16] Debye, P., Caulfield, D., and Bashaw, J., J. Chem. Phys. 41, 3051 (1964)

[17] Macedo, P. B., Simmons, J. H., and Haller, W., Phys. Chem. Glasses 9, 156 (1968).

[18| Simmons, J. H., Napolitano, A., and Macedo, P. B., J. Chem. Phys. 53, 1165 (1970).

[19| Simmons, J. H., and Macedo, P. B., J. Chem. Phys. 53, 2914 (1970).

[20] Haller, W., Blackburn, D. H., Wagstaff, F. E., and Charles, R. J., J. Am. Ceram. Soc. 53, 34 (1970).

[21] Napolitano, A., Macedo, P. B., and Hawkins, E. G., J. Res. NBS 69A, 449 (1965).

[22] Kawasaki, K., Phys. Rev. 150, 291 (1966); Kadanoff, L. P., Swift, J., Phys. Rev. 166, 89 (1968); Ferrell, R., Phys. Rev. Letters 24, 1169 (1970).

[23| Simmons, J. H., and Macedo, P. B., Conference on the Physics of Non-Crystalline Solids, Sheffield, England, September, 1970.

[24] Landau, L. D., and Lifshitz, E. M., Statistical Physics (AddisonWesley Publishing Co., Reading, Mass. 1958).

[25] Simmons, J. H., and Macedo, P. B., J. Chem. Phys. 54, 1325 (1971).

[26] Munster, A., Small Angle X-Ray Scattering, (Gordon and Breach, 1967) p. 401.

[27] Riebling, E. F., J. Am. Ceram. Soc. 47, 478 (1964).

[28 Hagy, H., private communication.

[29| Zarzycki, J., and Mezard, R., Phys. Chem. Glasses 3, 163 (1962).

(Paper 75A3-661) 